\title{
ONOMÁVAIN
}

Revista de lingüística, filología y traducción

\section{La explicitación en traducción o cómo traducir al español los eufemismos de tres obras clásicas chinas*}

The explicitation in translation or how to translate into Spanish the euphemisms of three Chinese classics

\section{Yazhi Cai \\ Universidad de Fudan \\ China}

\section{(c) $(i)$}

Yazhi Cai: Departamento de Filología Hispánica, Facultad de Lenguas y Literaturas Extranjeras, Universidad de Fudan, China. | E-mail:yazhicai@fudan.edu.cn 


\section{Resumen}

En este artículo, hemos procurado examinar la explicitación como un universal de traducción a través del estudio de la traducción al español de los eufemismos de tres obras clásicas chinas. Los resultados demuestran que la explicitación es una técnica de traducción recurrente en el corpus de textos literarios estudiado, lo que se corresponde con la hipótesis de explicitación planteada por varios teóricos; asimismo, mediante nuestro análisis, hemos podido comprobar que el grado de explicitación se vincula con el ámbito al que pertenece el eufemismo original y también está estrechamente relacionado con el estilo del traductor.

Palabras clave: explicitación; traducción literaria; eufemismo; universal de traducción; chino.

\section{Abstract}

In this article, we intend to examine the explicitation as a universal of translation through the translation into Spanish of euphemisms in three Chinese literary novels. The results indicate that explicitation is a frequently used technique of translation in the studied corpus of literary texts, which is consistent with the hypothesis of explicitation proposed by various theorists; in addition, through our analysis, we have been able to verify that the degree of explicitation is associated with the field of the original euphemism and is also related to the translation style of the translator.

Keywords: explicitation; literary translation; euphemism; translation universals; Chinese.

* Este estudio ha sido financiado por el Fondo del Ministerio de Educación de Ciencias Sociales de China (教育部人文社会科学研究青年基金项目“明清小说西译在西班牙语世界的传播与接受研究”) con número 18YJC740003. 


\section{Introducción}

Los estudios de traducción basados en corpus han proporcionado nuevas perspectivas y herramientas para los estudios descriptivos de traducción y han dado fructíferos resultados empíricos. A partir de los estudios de corpus, Baker (1993: 233-252) propuso cuatro universales de traducción que han sido ampliamente debatidos en las investigaciones posteriores. En el caso de la explicitación, que es uno de los universales más importantes según Baker, ya existen muchas investigaciones, aunque la mayoría estudian este universal desde un nivel macro, analizando elementos tales como la explicitación a través de conectores textuales en la traducción. Sin embargo, en la práctica descubrimos que la explicitación no solo es un fenómeno que se refleja en la cohesión textual. Así, nos preguntamos si podríamos verificar este universal en el nivel semántico. Por otra parte, al tratarse de un universal en traducción, ¿es posible que exista una correlación entre el grado de explicitación y otros factores tales como las características del texto y el estilo de cada traductor?

Basándonos en un estudio piloto que realizamos sobre la traducción al español del eufemismo en Hongloumeng (Sueño en el Pabellón Rojo), obra clásica china de la dinastía Qing (1636 d. C. - 1912 d. C.), logramos detectar la explicitación en la traducción de unos treinta y tres ejemplos de eufemismo, lo que constituye un material bastante limitado (Cai, 2015). Ahora bien, si ampliamos el estudio a un corpus mayor de obras y ejemplos, ¿seguirá existiendo este fenómeno?, ¿la explicitación estará relacionada con otros factores específicos? Estas son preguntas que pretendemos responder en este estudio.

\section{Eufemismo}

Puesto que en este estudio tratamos el eufemismo, consideramos que es importante aclarar a qué se refiere este recurso lingüístico. Según el DRAE (Diccionario de la Real Academia Española), el término eufemismo procede del lat. euphemismus, y este del gr. euphemismós; se trata en la definición académica de una "manifestación suave o decorosa de ideas cuya recta y franca expresión sería dura o malsonante”. Según Casas Gómez, quien ha desarrollado este asunto en su estudio La interdicción lingüística: mecanismos del eufemismo y disfemismo (1986), el eufemismo consiste en "una actualización discursiva por parte del hablante de unos sustitutos léxicos - habituales o lexicalizados u ocasionales o creativos- que, a través de un conjunto de recursos lingüísticos y paralingüísticos, permiten, en un contexto y situación pragmática determinada, neutralizar léxicamente el término interdicto" (Casas Gómez, 1986: 36). Asimismo, este autor es quien ha planteado en un esquema el mecanismo del eufemismo en una conferencia de 2009 titulada Hacia una nueva perspectiva del enfoque en la definición del eufemismo; según este autor, "ante una realidad interdicta, se hace una conceptualización, y mediante cambios léxicos se realiza una atenuación del término interdicto y la comunicación eufemística”. Por ejemplo, si nos referimos propiamente al "acto sexual", este se conceptualiza y da lugar a palabras como "cohabitar, copular, acostarse con alguien". 
De acuerdo con el esquema planteado por Casas Gómez, observamos que todos los eufemismos provienen de una realidad interdicta. El mecanismo no es uno y estático porque puede haber diferentes tipos de atenuación en la comunicación eufemística para una realidad interdicta, y podemos entender por qué existen tantas expresiones para una misma realidad y un referente original. Los hablantes pueden emplear en sus actos de habla sustitutos léxicos, sean habituales o lexicalizados, u ocasionales o creativos, como parte de la comunicación eufemística para neutralizar léxicamente el término interdicto, por lo que el eufemismo se convierte en un proceso de creación muy dinámico y presenta, además, una gran variedad. Por otra parte, como el eufemismo suele conllevar muchas connotaciones culturales propias de cada lengua y cultura, hecho que hemos analizado en un estudio anterior (Cai, 2019), y suele también tener un sentido implícito, su comprensión es a menudo complicada, por lo que constituye una dificultad de traducción en el proceso de la reverbalización en la lengua meta.

\section{Explicitación}

Vinay y Darbelnet (1995 [1958]: 342) mencionaron por primera vez la explicitación como un procedimiento de traducción y lo definen como "a stylistic translation technique which consists of making explicit in the target language what remains implicit in the source language because it is apparent from either the context or the situation". Nida (1964: 227-231) también profundizó en los conceptos de "explícito" e “implícito”, aunque los denominó de manera diferente. Para Nida, estos términos se refieren a técnicas de traducción cuya función es ajustar (adjustment) un texto traducido, especialmente los añadidos (additions), omisiones (subtractions) y cambios (alterations), entre los que subraya los añadidos, que vendría a equiparar con la explicitación. Según él, algunas de las razones por las cuales se utilizan los añadidos son: Ia necesidad de completar expresiones sobrentendidas, la obligatoriedad de especificar y los motivos de reestructuración gramatical, como, por ejemplo, la respuesta a preguntas retóricas, los elementos clasificadores y los conectores, entre otros. Como afirman Nida y Taber (1969: 33), "there is a tendency for all good translations to be somewhat longer than the originals. [...] It is almost inevitable”. Para Vázquez-Ayora (1977: 349), la explicitación es una "clase de expansión" con la que "se expresa en LM lo que está implícito en el contexto de la lengua origen”. En esta misma línea, Blum-Kulka (1986: 21) ha planteado la hipótesis de que la explicitación es una estrategia universal en traducción. Esta autora emplea conceptos de análisis del discurso para estudiar las explicitaciones relacionadas con los desplazamientos en los niveles de cohesión y coherencia y en los marcadores textuales de los textos traducidos. Además, en un estudio sobre la explicitación publicado en 1996, Kinga Klaudy presentó una clasificación tipológica de las explicitaciones: obligatorias, opcionales, pragmáticas y específicas de la traducción (translation-proper explicitation) (citado por Álvarez Lugris, 2001: 23).

Baker (1993), en un estudio de corpus titulado Corpus Linguistics and Translation Studies: Implications and Applications, propuso cuatro universales de la traducción, entre los que 
se encuentra el universal de la explicitación. Asimismo, en la Routledge Encyclopedia of Translation Studies (1998: 80-84), resume las ideas de otros autores sobre la "explicitación", cuya finalidad es ofrecer un texto meta lo más fiel posible y comprensible del texto origen para el receptor meta.

En China, Wang Kefei (2003) introdujo este término y publicó una serie de estudios. En su opinión, el fenómeno general de la explicitación puede ser una de las razones que llevan a la expansión del número de palabras del texto traducido; para ello, se centró en la explicitación de los pronombres personales. Otro investigador, Ke Fei (2005), cree que la investigación sobre la explicitación no debe limitarse a los cambios en la forma de la cohesión, sino que también debe estudiar la explicitación del significado y postula que la explicitación se ve influida por diversos factores tales como las características del idioma, los traductores y la cultura social. Además, Liu Zequan y Hou Yu (2008) creen que todavía queda mucho por estudiar en la investigación de este fenómeno.

En el caso de la traducción del eufemismo, la explicitación consiste en expresar el sentido implícito del eufemismo, lo cual se corresponde precisamente con lo que plantearon Vinay y Darbelnet (1995 [1958]: 342) y Blum-Kulka (1986: 21). Si el eufemismo pierde sus cualidades eufemísticas y resalta su significado implícito tras la traducción, entonces podemos comprobar que ha experimentado un proceso de explicitación en la traducción; en cambio, si el eufemismo aún mantiene su sentido eufemístico después de la traducción, diríamos que no ha experimentado una explicitación. Por ejemplo, cuando se traduce “仙游” (traducción literal: irse de viaje divino, eufemismo de "morir" en chino) por "ha muerto", se ha explicitado el sentido implícito. El eufemismo, por tener la característica particular de ser implícito, puede servir de material ideal para examinar este universal de la explicitación en la traducción.

Teniendo en cuenta lo que se ha investigado, creemos que los estudios existentes sobre la explicitación aún resultan insuficientes. Por ello, en el presente estudio, pretendemos profundizar en la traducción del eufemismo, figura cuya característica es tener significados implícitos, para analizar si el fenómeno de la explicitación se refleja a nivel semántico en la traducción del eufemismo y ver cómo sería el grado de explicitación y la correlación entre este último y otros factores. Además, los estudios ya existentes sobre la explicitación y el eufemismo se centran sobre todo en el inglés y todavía no se ha profundizado en el par de lenguas chino-español.

\section{Hipótesis de estudio}

Como hemos mencionado, en un estudio previo analizamos el fenómeno de la explicitación del eufemismo a partir de la traducción al español de Hongloumeng (Sueño en el Pabellón Rojo); ahora, diseñamos un nuevo estudio tomando como base nuevos materiales para verificar y profundizar en este fenómeno. Las hipótesis que planteamos en el presente trabajo son las siguientes: 
a) La explicitación constituye una técnica importante a la hora de traducir el eufemismo.

b) El grado de la explicitación tiene que ver con las características del texto original (las cuales están relacionadas con el ámbito al que pertenece el eufemismo) y también con el estilo de traducir de los traductores.

\section{Materiales y metodología}

A fin de probar nuestras hipótesis, hemos construido un corpus con una gran variedad de eufemismos extraídos de tres obras literarias chinas de las dinastías Ming (1368 d. C. - 1644 d. C.) y Qing (1636 d. C. - 1912 d. C.) y de sus traducciones al español. Las tres obras chinas y sus traducciones son:

a) 金瓶梅 Jin Ping Mei:

Traducción de Alicia Relinque Eleta. Jin Ping Mei (2010). Girona: Atalanta.

Es la primera versión traducida directamente del chino al español. A cargo de Alicia Relinque Eleta, basándose en el Jin Ping Mei Cihua, que es la versión más completa de esta obra.

b) 儒林外史 Rulin Waishi:

Traducción de Laureano Ramírez Bellerín. Los Mandarines (Historia del Bosque de los Letrados) (1991). Barcelona: Seix Barral.

c) 红楼梦 Hongloumeng:

Traducción de Zhao Zhengjiang y José Antonio García Sánchez. Sueño en el Pabellón Rojo (2010). Barcelona: Galaxia Gutenberg.

La razón por la cual hemos seleccionado estas tres obras y sus traducciones es, en primer lugar, que en ellas podemos encontrar una gran cantidad de eufemismos de diversos ámbitos. Además, puesto que las áreas predominantes de los eufemismos en cada obra son diferentes, podemos obtener suficiente material para el análisis de la traducción. En segundo lugar, con las tres traducciones podemos comparar y valorar los resultados obtenidos después de su análisis. Asimismo, podemos comparar los estilos de los traductores para su correspondiente discusión. Por último, estas tres novelas, como importantes obras literarias chinas, tienen gran valor artístico y nos permiten reflexionar desde la perspectiva de la traducción literaria. Las traducciones al español seleccionadas para este estudio se tradujeron directamente del chino al español.

Los eufemismos de las tres obras provienen de distintos ámbitos, entre los que destacan los referidos a la muerte, la sexualidad y el respeto. Extrajimos eufemismos de todas las variedades y aumentamos el número de ejemplos extraídos de aquellos eufemismos que se repitieron más veces. Tras el vaciado de los eufemismos de las tres novelas clásicas chinas, obtuvimos un total de 263 ejemplos, de los cuales 72 ejemplos fueron de Jin Ping Mei; 103 ejemplos, 
de Rulin Waishi, y 88 ejemplos, de Hongloumeng. Posteriormente, buscamos la traducción que se había hecho de cada uno de ellos, construyendo así un corpus paralelo. Sobre la base del análisis preliminar de la traducción, clasificamos todos los eufemismos obtenidos de la siguiente manera: capítulo, número de página, ámbito referencial del eufemismo, técnicas de traducción utilizadas, tendencia de la traducción (extranjerizante o domesticante) y si la traducción mantiene el mecanismo del eufemismo o no. Una vez terminado este proceso, analizamos las diferentes técnicas empleadas y las propuestas de traducción y sacamos las conclusiones.

Para realizar el estudio y clasificar las técnicas de traducción de los ejemplos, nos basamos en el esquema de Newmark (1992). Según su clasificación, las técnicas de traducción son: traducción literal, explicitación del sentido implícito, equivalente eufemístico, reducción, generalización, nota al pie de página, omisión, añadido, modulación y traducción mixta.

Asimismo, buscamos entrevistas hechas a los traductores para ver cómo afrontaban la traducción de obras chinas y consultamos algunos de sus artículos y libros sobre traducción. También nos sirvieron de referencia los prólogos de las traducciones de las tres obras chinas.

\section{Análisis}

A continuación, vamos a presentar el tipo de análisis que realizamos de los eufemismos seleccionados a partir de unos ejemplos representativos pertenecientes a los ámbitos de la muerte, la sexualidad, el respeto, los animales y el dinero. Después, presentaremos y discutiremos los resultados del análisis de todos los eufemismos extraídos de estas tres obras.

\section{TO: 我家老等没了一年有余,止有两位奶奶守寡,并不嫁人。(Capítulo 91, 《金瓶梅》)}

TM: Su señoría hace poco más de un año que falleció y sólo quedan dos esposas que le están guardando la viudedad, ninguna de las cuales tiene intención de casarse. (Tomo II, p. 1369, Jin Ping Mei)

La muerte es un ámbito donde aparecen numerosos eufemismos tanto en la cultura china como en la española. Muchos de ellos conllevan connotaciones culturales y religiosas y suelen tener una formulación compleja, pero, en este ejemplo, “没了” (traducción literal: desaparecer) es un eufemismo de uso convencional que apenas conlleva carga cultural; en la traducción, se ha explicitado con el verbo "fallecer", que originalmente era un eufemismo de la muerte pero que ha perdido su sentido eufemístico a lo largo de los años. Encontramos otros ejemplos semejantes de eufemismos de la muerte tales como “薨逝” (traducción literal: oscurecerse la mirada e irse), “亡故” (traducción literal: fallecer), “不中用了” (traducción literal: no servir para nada), “不在了” (traducción literal: dejar de estar), “仙游” (traducción literal: emprender un viaje divino), “见背” (traducción literal: irse), “寿终” (traducción literal: terminar la vida), “去世” (traducción literal: despedirse del mundo), etcétera. En la mayoría de los casos, 
estos eufemismos se explicitaron en la traducción, pues no tienen demasiada carga cultural. No obstante, algunos no fueron explicitados por los traductores; por ejemplo, “状天” (traducción literal: volver al cielo) se tradujo como "ha vuelto al cielo", ya que es un eufemismo que transmite cierta connotación religiosa.

TO: 第二件,我小时在三街两巷游串,也曾养得好大龟。(Capítulo 3, 《金瓶梅》)

TM: En cuanto a la segunda, ya desde chico recorrí callejas y barrios de placer, y alimenté una tortuga de hermoso tamaño. (Tomo I, p. 139, Jin Ping Mei)

La sexualidad también es un ámbito recurrente del eufemismo. En este ejemplo, la tortuga es un eufemismo formado mediante una metáfora que ha utilizado el personaje Ximen Qing para referirse al pene, y, en la traducción, se ha usado la técnica de la traducción literal, manteniendo así la metáfora consistente en comparar el pene con una tortuga. Puesto que en la cultura española no se suele usar la tortuga para referirse al pene, la traductora soluciona este problema añadiendo una nota a pie de página: “la «tortuga» (gui) es un eufemismo de "pene»". De esta manera, se conserva la formulación original y al mismo tiempo, mediante la nota, los lectores pueden comprender el sentido implícito de esta expresión. En este ejemplo, observamos que el eufemismo no ha experimentado una explicitación. Veamos otro ejemplo del mismo ámbito referencial de la sexualidad:

TO:这贾琏,虽然应名来上学, 亦不过虚掩耳目而已。仍是斗狗走鸡, 赏花玩柳。(Capítulo 9, 《红楼梦》)

TM: Pero su asistencia no era sino la fachada que escondía su acendrada afición a las peleas de gallos, las carreras de galgos y los burdeles. (Tomo I, p. 181, Hongloumeng)

“赏花玩柳” (traducción literal: apreciar las flores y divertirse con los sauces). En el caso de haberse hecho una traducción literal, los lectores muy probablemente no hubieran comprendido el sentido del To; por eso, el traductor traduce "los burdeles", explicitando así el sentido oculto del eufemismo chino. Con esta solución, se pierde la forma eufemística pero se transmite el sentido original. Encontramos muchos eufemismos de este ámbito en Jin Ping Mei y en Hongloumeng. Por ejemplo, existen otras expresiones como “枕边风月” (traducción literal: viento y luna al borde de la almohada), “颠变倒凤” (traducción literal: fénix y dragón enlazados y revueltos), “鱼水之欢” (traducción literal: alegrías de los peces en el agua), “沾风 惹草” (traducción literal: acariciar el viento y provocar a la hierba), “云雨” (traducción literal: nube y Iluvia), “飘风戏月” (traducción literal: volar con el viento y coquetear con la luna), “春 意” (traducción literal: deseos primaverales), etcétera. Las formulaciones son muy peculiares en comparación con el español y utilizan diversas metáforas. En este caso, para conservar en la traducción las características de la formulación original, se recomienda aplicar la técnica de la traducción literal; por otra parte, aunque esos eufemismos tienen formulaciones que no existen en la cultura española, el contexto y la nota a pie de página facilitan la comprensión del sentido implícito a los lectores. 
A continuación, comentaremos algunos ejemplos del ámbito del respeto. Los eufemismos en chino de este ámbito suelen añadir un término elogioso delante del referente para ensalzar la posición del interlocutor, o añadir un término devaluativo para rebajar la posición de uno mismo. Por eso, para referirse al interlocutor, en vez de emplear el tuteo, existen las expresiones “老先生” (traducción literal: maestro), “长兄” (traducción literal: hermano mayor), “贤契” (traducción literal: joven virtuoso), “尊驾” (traducción literal: estimada carroza), que destacan la sabiduría o la virtud del interlocutor. En las conversaciones también encontramos otro tipo de eufemismos para rebajar la posición de uno mismo y mostrar modestia; las estructuras más frecuentes son: “粫” (humilde) + X, “拙” (torpe) + X, “老” (viejo) + X, “寒” (lamentable) + X, “晚生” (menor en edad), “小的” (traducción literal: el pequeño yo), “贱” (despreciable) + X, “患” (estúpido) + X, “虚” (traducción literal: en vano) + X, “小”(pequeño) + X, etcétera. En la obra Rulin Waishi, que es un retrato fiel del círculo de los intelectuales de la época en que se publicó durante la dinastía Qing, existe una gran cantidad de eufemismos de este ámbito. Para referirse a uno mismo, en lugar de nombrarse directamente utilizando el pronombre "yo", hay diversas expresiones para rebajar la propia posición con el objetivo de mostrar modestia: por ejemplo, la expresión “小弟” significa literalmente “hermano menor” y se usa para referirse a uno mismo cuando se tiene menos edad o una posición social más baja. En cuanto a su traducción, encontramos las siguientes formas:

a) "un servidor" (equivalente eufemístico):

TO: 小弟站在彩棚门口 (Capítulo 4, 《儒林外史》)

TM: y un servidor aguardó a su puerta. (p. 65, Rulin Waishi)

b) “vuestro hermano menor" (traducción literal):

TO: 小弟年幼 (Capítulo 7, 《儒林外史》)

TM: Vuestro hermano menor es joven. (p. 103, Rulin Waishi)

c) “yo" (explicitación):

TO: 小弟从实说: 不曾认得。(Capítulo 4, 《儒林外史》)

TM: Yo contesté con toda honradez: No. (p. 66, Rulin Waishi)

Para traducir la expresión “小弟” (traducción literal: hermano menor), se han dado diferentes traducciones en cada uno de los tres ejemplos: en a) se ha traducido por un equivalente en español, "un servidor"; en b) se ha traducido literalmente, y en c) se ha explicitado el "yo". Esto nos muestra que pueden existir distintas opciones para traducir una misma expresión, como sucede en estos tres casos en los que se ha optado por tres técnicas diferentes.

Presentamos también dos eufemismos de los ámbitos relacionados con los animales y las actividades económicas.

TO: 天色已黑了,倘或又跳出一个大虫来,我却怎地斗得他过? (Capítulo 1, 《金瓶梅》)

TM: Ya es de noche, si aparece otro tigre no podré con él. (Tomo I, p. 94, Jin Ping Mei) 
“大虫” (traducción literal: bicho grande) es otro modo de denominar a un tigre; este eufemismo proviene de la Dinastía Tang (618-917 d. C.), ya que el abuelo del primer emperador Li Yuan se llamaba Li Hu, y Hu significa tigre. Para evitar vincular el tigre con su nombre, por el tabú de no mencionar directamente los nombres de los antepasados, se crearon expresiones como 亚 龙 (traducción literal: dragón de segundo rango), 威物 (animal poderoso) y 大虫 (bicho grande). En la traducción, vemos que se ha explicitado el sentido implícito.

Las actividades económicas son un ámbito en el que se encuentran muchos eufemismos de la vida cotidiana hoy en día, dentro de los cuales encontramos las expresiones “打秋风” (traducción literal: pedir el viento del otoño), “宦囊” (traducción literal: bolsillo de un oficial) y “所 以然” (traducción literal: de aquella manera).

TO: 张世兄屡次来打秋风甚是可厌 (Capítulo 4, 《儒林外史》)

TM: El amigo Zhang ya ha venido hartas veces a pedirme dinero, y no hay quien lo sufra. (p. 67, Rulin Waishi)

“打秋风” se entiende literalmente por “pedir el viento del otoño” y se ha derivado desde la expresión “打秋丰” por la homofonía del “风” (viento) y “丰” (cosecha), que significa originalmente "pedir la cosecha del otoño" y alude a las personas que vienen para aprovecharse de las propiedades de los otros usando sus relaciones personales. En la traducción, se ha explicitado la idea de "pedir dinero".

\section{Resultados del análisis de los eufemismos extraídos}

En los ejemplos anteriores, podemos comprobar cómo los traductores han optado en muchas ocasiones por la técnica de la explicitación para traducir el eufemismo. En nuestra opinión, este hecho puede deberse a diferentes motivos.

Durante la traducción, en la búsqueda de un equivalente comunicativo, cabe la posibilidad de elegir otro término ya existente en la lengua de llegada que sustituya o atenúe el original; pero si ese término no existe en la cultura meta, el traductor ha de recurrir al referente original o crear un término nuevo. Ahora bien, si el traductor solo mantiene la forma original del eufemismo, los lectores de la cultura meta no entenderán exactamente qué quiere decir y, por ello, se suele optar por la técnica de la explicitación. Otra razón para explicar esta técnica es que, en algunas ocasiones, cuando no se trata de un eufemismo que tiene una formulación peculiar ni una connotación cultural muy grande, al leerlo el traductor capta su sentido implícito muy fácilmente pero no su función eufemística, por lo que lo explicita inconscientemente en la traducción. Eso ocurre con muchos eufemismos de uso convencional que no tienen connotaciones culturales. 


\subsection{Grado de explicitación}

Creemos que la explicitación frecuentemente constituye un ejemplo de las muchas apreciaciones impresionistas y no rigurosas que se han hecho sobre la traducción. Además, como hemos mencionado, el eufemismo es un tipo de material ideal para examinar la hipótesis de la explicitación. Basándonos en el análisis de todos los ejemplos extraídos de las tres obras, encontramos que de los 263 ejemplos se han explicitado 78 casos (28\%), lo que constituye un porcentaje bastante alto; por lo tanto, hemos podido comprobar que la explicitación es una forma de traducción relativamente frecuente en la traducción del eufemismo de las tres obras.

\section{GRÁFICO 1}

Explicitación global de la traducción al español del eufemismo en las tres obras

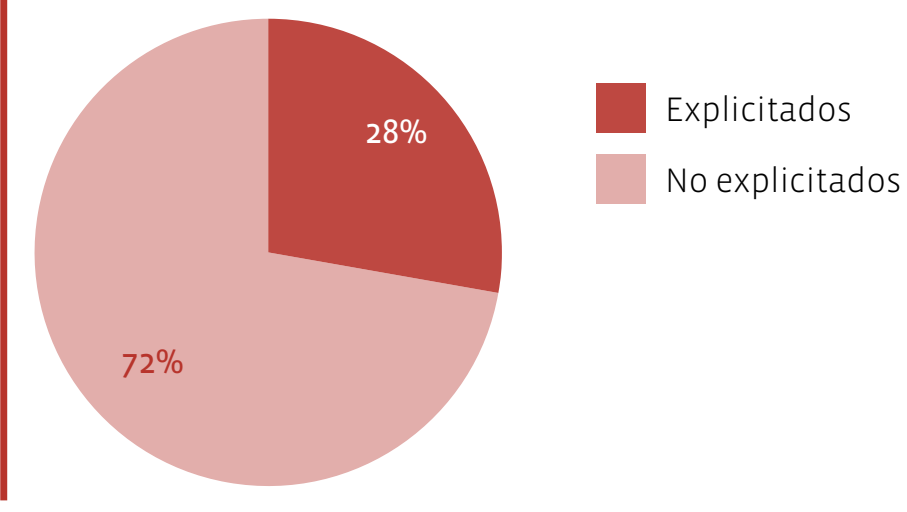

Sin embargo, observamos distintos grados de explicitación en la traducción de los eufemismos de las tres obras. Como se ve en el gráfico 2, en Jin Ping Mei, en total se ha explicitado un $13 \%$ de los eufemismos; en Rulin Waishi, se ha explicitado un 32\%, y, en Hongloumeng, se ha explicitado un $34 \%$.

\section{GRÁFICO 2}

Grado eufemístico tras la traducción del eufemismo
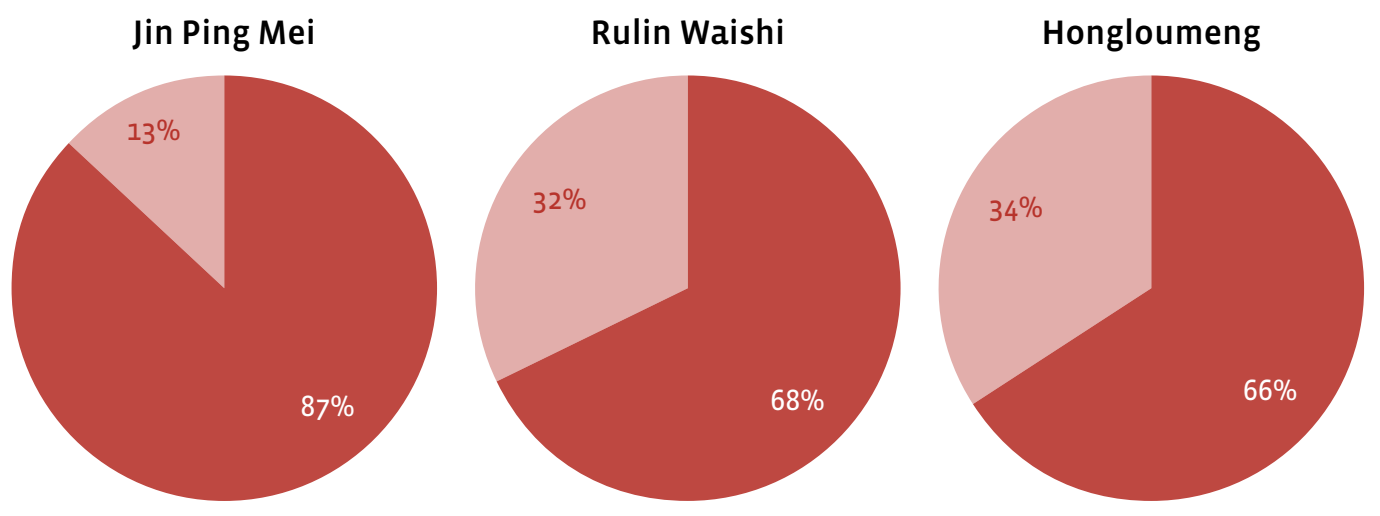

Eufemístico

No eufemístico 
El fenómeno de la explicitación corresponde a uno de los cuatro universales que planteó Baker y podemos confirmar que a nivel semántico sí existe una explicitación evidente. Aun así, no podemos tampoco negar que en algunos casos el mismo significado del eufemismo puede resultar igualmente intrincado y más complicado de comprender para los lectores.

Por otra parte, hemos averiguado que el grado de explicitación puede variar dependiendo del texto original. A continuación, vamos a ver cómo el tipo de texto influye en el grado de explicitación.

\subsection{Explicitación y características del eufemismo original}

En el estudio, encontramos que existen ciertas correlaciones entre el grado de explicitación y varios factores. Primero, el grado de explicitación en la traducción está vinculado con las características del texto original y el ámbito al que pertenece el eufemismo.

Como sabemos, cada una de las obras tiene diversos eufemismos en diferentes ámbitos y los eufemismos de cada ámbito suelen tener características estructurales peculiares; además, los eufemismos correspondientes en español tienen también características diferentes. Esas diferentes características estructurales del eufemismo afectan en cierta medida al grado de explicitación en la traducción.

\section{TABLA 1}

Grado de explicitación de los eufemismos de distintos ámbitos

\begin{tabular}{lccc} 
& JIN PING MEI & RULIN WAISHI & HONGLOUMENG \\
Sexualidad & $0 / 32=0$ & $0 / 0=0$ & $7 / 24=29 \%$ \\
\hline Respeto y modestia & $6 / 25=24 \%$ & $21 / 60=35 \%$ & $4 / 12=33 \%$ \\
\hline Muerte & $4 / 7=57 \%$ & $8 / 18=44 \%$ & $15 / 43=35 \%$ \\
\hline
\end{tabular}

Como se ve en la tabla 1, de todos los ámbitos referenciales, el eufemismo del ámbito de la muerte se ha explicitado con un grado más alto en las traducciones de las tres obras. Es decir, una vez traducidos, una gran parte de los eufemismos de este ámbito han perdido su significado eufemístico. Creemos que la razón más probable es que los eufemismos de muerte más usados suelen ser menos eufemísticos en su construcción original, por lo que el traductor puede fácilmente ignorar su formulación eufemística cuando lee el texto original. Por otro lado, la construcción del eufemismo del ámbito de la muerte conlleva relativamente una menor connotación cultural y por ello no atrae especialmente la atención de los traductores. Por lo tanto, los eufemismos de este tipo a menudo se traducen por una expresión que no tiene un sentido eufemístico. Por ejemplo, “亡” (traducción literal: fallecer) y “去世” (traducción literal: irse del mundo) son eufemismos de “死” (morir), pero, cuando el traductor los lee, es fácil equiparar inconscientemente el significado de “亡”" con “死”. Por todo ello, estos eufemismos 
se han explicitado en la traducción y el grado de explicitación es más alto. Aún así, cabe tener en cuenta que también existen algunos eufemismos de formulación especial cuyas connotaciones religiosas son evidentes y no se han explicitado en la traducción.

Al contrario que los eufemismos sobre la muerte, en el ámbito de la sexualidad, el grado de explicitación es el más bajo tanto en Jin Ping Mei como en Hongloumeng. En el contexto de la literatura clásica china, estos eufemismos se relacionan a menudo con los elementos de la naturaleza, como el viento, la luna, las flores, la hierba, el sauce y otros elementos asociados a la feminidad. Esta formulación es muy especial en comparación con la cultura española; además, para su composición, a menudo se utilizan diferentes procedimientos semánticos como la metáfora y el cultismo. En el proceso de la traducción, es fácil que este tipo de eufemismos atraiga la atención de los traductores, y, para preservar su significado literal y su expresión literaria, estos tienden a utilizar la traducción literal. Además, para que el lector conozca su significado oculto, los traductores suelen añadir notas.

En la traducción de los eufemismos del ámbito del respeto y la modestia, encontramos que la estrategia más utilizada es la traducción literal. En las tres obras analizadas, los eufemismos de este ámbito frecuentemente se componen de ciertas estructuras fijas. En chino se agregan adjetivos como “贵” (vunerable) o “贱” (despreciable) antes de la referencia, como “贵姓” (apellido vunerable) y “尊名” (nombre respetable). Para reflejar las características de la expresión original, la mayoría de las traducciones tienden a conservar esta estructura original. Por lo tanto, debido al uso frecuente de la traducción literal, su grado de explicitación no es tan alto. Además, también hay algunas expresiones eufemísticas equivalentes en español, como "vuestra merced", "vos" y otras por el estilo. Todo ello provoca que haya menos grado de explicitación en el eufemismo del respeto que en el eufemismo de la muerte.

En resumen, las características del texto original son un factor muy relevante de la explicitación, es decir, podemos señalar que existe una tendencia: cuanto más marcado culturalmente sea el eufemismo del texto original, más puede captar la atención del traductor y menor es la explicitación en la traducción.

Esto puede también explicar las diferencias en el grado de explicitación del eufemismo en las tres obras. Dado que el ámbito del eufemismo influye en el grado de explicitación, este se ve determinado por la diferente distribución de los ámbitos en las tres novelas. Como hemos analizado, en el ámbito del sexo es más difícil encontrar en español un equivalente eufemístico y se usa más la traducción literal para no perder la formulación original y la carga cultural que lleva el eufemismo. Sin embargo, en el ámbito de la muerte, la técnica más utilizada es el equivalente eufemístico y, en el ámbito del respeto, la traducción literal y el equivalente eufemístico. De todas estas técnicas, la traducción literal está más ligada a la tendencia extranjerizante, mientras que el equivalente eufemístico y la explicitación se relacionan más con la tendencia domesticante. 


\subsection{La correlación entre la explicitación y los traductores}

No faltan las metáforas referidas a la labor de los traductores: traducir es "bailar con las cadenas en los pies", que resalta la dificultad de la traducción, o traducir es "pintar un cuadro" reflejando el paisaje original pero utilizando su propio color, es decir, utilizando sus propios estilos y características. Estas metáforas muestran cómo las características particulares de los traductores influyen en sus traducciones. Además de las características del texto original y del ámbito al que pertenece el eufemismo, encontramos que el grado de la explicitación también está estrechamente relacionado con el método y objetivo del traductor. Cuando la tendencia domesticante del traductor es más obvia, el grado de explicitación de su traducción es mayor.

En el prólogo de la traducción de Jin Ping Mei, la traductora Alicia Relinque señala que su objetivo es intentar ser lo más fiel posible. En un artículo titulado "Cómo la traducción transforma el aspecto de la literatura", Relinque (2013: 159-163) insiste en esta idea y valora precisamente el extrañamiento producido al realizar una traducción extranjerizante, lo cual se corresponde con la concepción de Viktor Shkolvsky (1893-1984) sobre el arte y la literariedad: la literatura es un tipo de texto que nos produce una extrañeza estética que nos aleja de la vida diaria:

The purpose of art is to impart the sensation of things as they are perceived and not as they are known. The technique of art is to make objects "unfamiliar", to make forms difficult, to increase the difficulty and length of perception because the process of perception is an aesthetic end in itself and must be prolonged. Art is a way of experiencing the artfulness of an object: the object is not important [...] (Viktor Shkolvsky, 1965: 12).

En esa línea, la traductora presta mucha atención al contenido original, ya que en la traducción de las obras clásicas es clave mantener los elementos culturales con objeto de acercar la lengua china a los lectores o bien con fines académicos, puesto que Jin Ping Mei es una obra que inspira muchas investigaciones. Así pues, la traductora busca provocar en el lector un extrañamiento usando la tendencia extranjerizante. En su traducción, los eufemismos aparecen explicitados en un grado muy bajo, ya que optó por conservar en la mayoría de los casos las formulaciones peculiares de la lengua original.

Por su parte, el traductor Zhao Zhengjiang ha destacado las dificultades que tuvo a la hora de traducir Hongloumeng. Según él, la traducción puede construir un puente entre dos culturas. En este sentido, hay que intentar que el contenido se entienda de manera clara para los lectores a la vez que estos se dan cuenta de la importancia de la obra y de la necesidad de mantener las expresiones peculiares del texto original (citado por Zhongguowang, 2008). En cuanto al traductor de Rulin Waishi, Laureano Ramírez, no ha manifestado explícitamente sus objetivos, pero observamos que da especial énfasis a la creatividad del traductor en el proceso de traducir (Ramírez, 1999: 86), especialmente por tratarse de dos idiomas tan lejanos 
como el chino y el español. Por lo tanto, en su traducción procura la creatividad a la hora de buscar equivalentes, para lo cual emplea los recursos retóricos más apropiados de la lengua de llegada. Ramírez señala también la importancia de mantener “la misma fuerza expresiva” en la lengua meta. Esto se corresponde con la postura de Nida sobre la equivalencia dinámica, que consiste justamente en provocar el mismo efecto en los lectores meta que tuvieron los lectores de la lengua de origen. Por todo ello, en las traducciones de Hongloumeng y Rulin Waishi, los traductores enfatizan más la recepción comunicativa de los lectores, por lo que han explicitado en un grado relativamente más alto los eufemismos.

Podemos decir que, en general, entre las tendencias de la extranjerización y de la domesticación, los traductores han adoptado posturas diferentes. Alicia Relinque Eleta tiende claramente a la extranjerización y explicita menos, mientras que Laureano Ramírez Bellerín y Zhao Zhenjiang se decantan por la domesticación. Como hemos señalado, el grado de explicitación de la tendencia extranjerizante es relativamente más bajo que el de la domesticante.

\section{Conclusiones}

En este estudio hemos investigado la traducción del eufemismo a partir de los ejemplos seleccionados de tres obras literarias clásicas chinas (Jin Ping Mei, Rulin Washi y Hongloumeng) y sus traducciones en español. Este material nos ha servido para examinar desde el nivel semántico la hipótesis de la explicitación en traducción, la cual fue planteada por varios teóricos. A través del vaciado, clasificación y análisis de un total de 263 ejemplos, hemos podido ver que, a la hora de traducir los eufemismos de diversos ámbitos referenciales (muerte, sexualidad, respeto, actividades económicas, etcétera), la explicitación constituye una de las técnicas predominantes de traducción en el corpus estudiado, pues se ha detectado un porcentaje relativamente alto de explicitación en dicho corpus.

En cuanto a los factores relacionados con el grado de explicitación de los eufemismos, hemos visto que las diferencias se deben a dos factores: por un lado, a las características del texto original y, por otro lado, a la tendencia de traducción y el método de los traductores. Así, en relación con lo primero, se comprueba una tendencia a traducir con una mayor o menor explicitación según el ámbito referencial, del cual depende a su vez la formulación del eufemismo. Por ejemplo, en el ámbito de la muerte, el grado de explicitación en la traducción es relativamente más alto, mientras que en el de la sexualidad dicho grado es mucho más bajo. En cuanto al segundo factor que influye en el grado de explicitación, cabe destacar que los traductores de tendencia extranjerizante suelen explicitar menos. Con todo ello, podríamos concluir señalando que el presente trabajo ayuda a completar los estudios sobre los universales de la traducción, en concreto, sobre el universal de la explicitación, y aporta a su vez reflexiones sobre la traducción literaria entre un par de lenguas tan lejanas como son el chino y el español. 


\section{Bibliografía citada}

Álvarez Lugris, Alberto, 2001: "Hipótesis de explicitación: ¿universal de la traducción o tendencia? Estudio sobre el corpus tectra de traducciones del inglés al gallego" en Anne BarR, M. Rosario Martín Ruano y J. Torres del Rey (eds.): Últimas corrientes teóricas en los estudios de traducción y sus aplicaciones, Salamanca: Ediciones Universidad de Salamanca, 22-33.

BAKER, Mona, 1993: "Corpus Linguistics and Translation Studies: Implications and Applications" en Mona Baker, Gill Francis y Elena Togninl-Bonelu (eds.): Text and Technology. In honour of John Sinclair, Amsterdam: Benjamins, 233-252.

BAKER, Mona, 1998: Routledge Encyclopedia of Translation Studies, London / New York: Routledge.

BLum-Kulka, Shoshana, 1986: "Shifts of Cohesion and Coherence in Translation" en Juliane HouSE y otros (eds.): Interlingual and Intercultural Communication: Discourse and Cognition in Translation and Second Language Acquisition Studies, Tübingen: Gunter NarrVerlag, 17-35.

CAl, Yazhi, 2015: "La traducción del eufemismo del chino al español: Hongloumeng y su traducción Sueño en el Pabellón Rojo", Hikma 14, 37-54.

CAl, Yazhi, 2019: "Estudio comparativo del eufemismo en chino y español", Círculo de Lingüística Aplicada a la Comunicación 77, 3-20.

CAo, Xueqin, 2010: Sueño en el Pabellón Rojo, traducción de Zhao Zhenjiang y José Antonio Garcia Sanchez, edición revisada por Alicia Relinque Eleta, Barcelona: Galaxia Gutenberg.

CAO, Xueqin [曹雪芹], 2002: 红楼梦, 北京: 人民文学出版社. (Hongloumeng, Beijing: Renmin Wenxue Chubanshe).

Casas Gómez, Miguel, 1986: La interdicción lingüística: Mecanismos del eufemismo y el disfemismo, Cádiz: Universidad de Cádiz.

Casas Gómez, Miguel, 2009: "Hacia una nueva perspectiva del enfoque en la definición del eufemismo" en Catalina Fuentes Rodriguez y Esperanza R. Alcaide Lara (coords.): Manifestaciones textuales de la descortesía y agresividad verbal en diversos ámbitos comunicativos, Sevilla: Universidad Internacional de Andalucía, 11-29.

LIu, Zequan [刘泽权], y Hou Yu [侯羽], 2008: “国内外显化研究现状概述”, 《中国翻译》5, 55-58. (“Descripción global de las investigaciones sobre la explicitación”, Traducción China.)

NIDA, Eugene, 1964: Toward a Science of Translating: With Special Reference to Principles and Procedures Involved in Bible Translating, Leiden: E. J. Brill. 
NidA, Eugene, y Charles TABER, 1969: The theory and practice of translation, Leiden: E. J. Brill.

VÁzquez-Ayora, Gerardo, 1977: Introducción a la Traductología, Washington D. C.: Georgetown University Press.

Vinay, Jean-Paul, y Jean Dalbernet, 1995 [1958]: Comparative Stylistics of French and English: A Methodology for Translation, traducido y editado por Juan C. SAGER y M.-J. HameL, Amsterdam y Filadelfia: John Benjamins Publishing Company.

El erudito de las carcajadas, 2010: Jin Ping Mei en verso y en prosa, traducción de Alicia Relinque ELETA, Girona: Atalanta.

EL ERUDITO DE LAS CARCAJADAS [兰陵笑笑生], 2010:《金瓶梅词话梦梅馆校本》, 台北: 里仁书局. Uin Ping Mei Ci Hua de la versión Mengmei, Taibei: Liren Shuju.)

KE, Fei [柯飞], 2005: “翻译中的隐和显”, 《外语教学与研究》4, 303-307. “"La explicitación e implicitación en la traducción”, Enseñanza e investigación en lenguas extranjeras.)

KLAudy, Kinga, 1996: "Back-Translation as a Tool for Detecting Explicitation Strategies in Translation” en Kinga KLaudy, José Lambert y Anikó SohÁr (eds.): Translation Studies in Hungary, Budapest: Scholastica Kiadó, 80-84.

Newmark, Peter, 1992: Manual de traducción, traducción de Virgilio Moya, Madrid: Cátedra.

Ramírez Bellerín, Laureano, 1999: Del carácter al contexto. Teoría y práctica de la traducción del chino moderno, Barcelona: Universitat Autónoma de Barcelona.

Real Academia Española: Diccionario de la lengua española [http://lema.rae.es/drae/?val=eufe mismo] [fecha de la última consulta: 15 de febrero de 2019].

Relinque Eleta, Alicia, 2010: “Prólogo” en El erudito de las carcajadas: Jin Ping Mei en verso y en prosa, traducido por Alicia Relinque Eleta, Girona: Atalanta.

Relinque EleTA, Alicia [雷琳克], 2013: “操斧伐柯:论翻译如何改变文学的面貌”, 《世界汉学》12, 159-164. (“Tomar un hacha para tallar un mango: cómo la traducción transforma la literatura”, Sinología Mundial.)

Shkolvsky, Viktor, 1965: “Art as Technique” en Lee T. Lemon y Marion J. Reiss (eds.): Russian Formalist Criticism: Four Essays, Lincoln: University of Nebraska Press, 17-23.

WANG, Kefei [王克非], y Libo HuANG [黄立波], 2007: “语料库翻译学的几个术语”, 《四川外语学院 学报》6, 101-105. (“Algunos términos en los estudios de traducción basados en corpus”, Revista de la Universidad de Lenguas Extranjeras de Sichuan.) 
Wu, Jingzi, 2007: Los Mandarines: Historia del Bosque de los Letrados, presentación, traducción del chino y notas por Laureano Ramírez Bellerin, Barcelona: Seix Barral.

Wu, Jingzi [吴敬梓], 1977: 《儒林外史》, 北京: 人民文学出版社. (Rulin Waishi, Beijing: Renmin Wenxue Chubanshe.)

ZHONGGUOWANG, 2008: “对话翻译家: 赵振江与《红楼梦》” “Diálogo con el traductor: Zhao Zhenjiang y Hong/oumeng”) [http://webcast.china.com.cn/webcast/created/2163/44_1_0101_desc. htm] [fecha de la última consulta: 20 de enero de 2019]. 